\title{
Aerostructural Design Optimization of an Adaptive Morphing Trailing Edge Wing
}

\author{
David A. Burdette * \\ Gaetan K.W. Kenway ${ }^{\dagger}$ \\ Zhoujie Lyu $\ddagger$ \\ Joaquim R. R. A. Martins ${ }^{\S}$ \\ University of Michigan, Ann Arbor, Michigan, 48109, United States
}

\begin{abstract}
Adaptive morphing trailing edge technology offers the potential to decrease the fuel burn of transonic transport aircraft by allowing wings to dynamically adjust to changing flight conditions. Current aircraft use flap and aileron droop to adjust the wing during flight. However, this approach offers only a limited number of degrees of freedom, and the gaps in the wing created when using these devices introduce unnecessary drag. Morphing trailing edge technology offers more degrees of freedom, with a seamless interface between the wing and control surfaces. In this paper we seek to quantify the extent to which this technology can improve the fuel burn of transonic commercial transport sized aircraft. Starting from the undeformed Common Research Model (uCRM) geometry, we perform fixed-planform aerostructural optimizations of a standard wing, a wing retrofitted with a morphing trailing edge, and a clean sheet wing designed with the morphing trailing edge. The wing retrofitted with the morphing trailing edge improved the fuel burn as effectively as the full wing redesign without morphing. Additional fuel burn reductions were observed for the clean sheet design. The morphing trailing edge decreased the fuel burn by performing load alleviation at the maneuver condition, weakening the trade-off between cruise performance and maneuver structural constraints, resulting in lighter wingboxes and more aerodynamically efficient cruise configurations.
\end{abstract}

\section{Introduction}

Aircraft efficiency has attracted increased attention in recent years due in large part to increasing fuel prices and concerns about the environmental impacts of the airline transportation industry. This increased consideration of aircraft efficiency has translated to increased research on ways to reduce fuel burn, which has produced a number of fuelsaving approaches. One such approach is the use of trailing edge deflections to tailor the wing shape to specific flight conditions along its trajectory. Several modern aircraft use this approach, utilizing flap and aileron droop to alter the wing camber depending on the flight condition and the aircraft's weight. While this implementation of trailing edge morphing improves the wing's efficiency, further improvements could be realized through the use of continuous morphing trailing edge technology. This technology could remove losses due to the control surface seams, while also giving the control surfaces additional degrees of freedom. These devices have been research topics for some time, but the hardware has recently taken substantial strides towards market readiness, as evidenced by the FlexSys FlexFoil $[1,2]$. As this technology is nearing commercial availability, it is important to quantify how significant its effects on aircraft efficiency will be.

There have been many morphing trailing edge studies which have been centered around development of the hardware necessary for physical morphing wing technology $[1,2,3]$. There have also been aerodynamic studies of morphing trailing edges [4]. However, these studies used low-fidelity models that do not accurately capture the effects of small geometric deformations. Higher-fidelity RANS models have shown that very small geometric changes can have substantial effects on the flow characteristics [5]. Lyu and Martins [6] performed high-fidelity aerodynamic optimization of a morphing trailing edge on the CRM wing and achieved a 1-5\% drag reduction. That work considered only the aerodynamics of the wing, while assuming a constant 1-g deflected wing profile. Neglecting structural effects produced wings at various flight conditions that were close to their optimized shapes. As such, there were limited improvements available for the morphing trailing edge. Including coupling between the aerodynamics and structural deformations produces wings that show much larger changes as a result of the different flight conditions. It follows

\footnotetext{
*Ph.D. Candidate, Department of Aerospace Engineering, AIAA Student Member

$\dagger$ Post Doctoral Research Fellow, Department of Aerospace Engineering

$\ddagger$ Ph.D. Candidate, Department of Aerospace Engineering, AIAA Student member

$\S$ Associate Professor, Department of Aerospace Engineering, AIAA Associate Fellow
} 
then that there should be larger gains possible through the use of morphing trailing edges when accounting for the wing flexibility and performing aerostructural optimization.

To investigate the effectiveness of morphing trailing edge devices, we perform a series of aerostructural optimizations on the uCRM geometry [7]. This geometry was developed to closely match the undeflected geometry of the CRM case, for which the only provided geometry represents the deflected 1-g shape. Aerostructural optimization with our tools requires the wing's undeflected jig shape. We consider a number of comparisons between various optimizations in order to develop an understanding of the effectiveness of these devices.

This work is outlined as follows: Section II summarizes the software tools used to perform the aerostructural optimization. Section III provides the details of the formulation of the optimization problem. Section IV discusses the aerostructural optimization results. Finally, in Section V we conclude and consider future work related to this topic.

\section{Software Overview}

In this section we described computational tools used for various aspects of the aerostructural analysis and optimization within the multidisciplinary design optimization of aircraft configurations with high fidelity (MACH) framework [8]. This framework has been used previously to perform aerostructural optimization of the uCRM geometry [7], as well as other investigations $[9,10,11]$.

\section{A. Geometric Parametrization}

The aircraft geometry is parametrized using a free-form deformation (FFD) [12] approach. In this FFD approach, the aircraft is embedded within an FFD volume, which is then deformed using a number of control points. This parametrization reduces the number of wing shape design variables. Through relatively simple manipulations of the control points, complex geometric designs can be achieved. Control points can also be grouped to create full-wing design variables such as span, chord, and sweep. Another benefit of parametrizing the geometry using an FFD is that deformations of the structural mesh and aerodynamic surfaces are both handled the same way, meaning that no additional effort is needed to deform the internal structure of the wing to match the optimizer's changes to the aerodynamic surfaces. An example of an FFD around a wing is shown in Figure 2.

\section{B. Mesh Deformation}

As the geometry of the aircraft is altered through structural deformations and design variable changes, the mesh is required to adjust appropriately. It is important that the quality of the deformed mesh at each of the optimization iterations remains high enough to provide reliable results. However, manual input within optimization iterations are prohibitively slow, and in general this is a poor practice. As such, this process needs to be automated. In this work, the mesh deformation was completed using an inverse distance weighting interpolation warping algorithm [13], which interpolates node translations and rotations to help preserve orthogonality of boundary layer meshes. This is particularly important in this work given the substantial rotations produced by the morphing trailing edge.

\section{Aerodynamic Solver}

The CFD code used within MACH is SUmb [14], a parallel, finite-volume, cell-centered, multi-block solver. In order to sufficiently resolve the flow around the aircraft and to achieve the fidelity needed to effectively quantify the benefits of a morphing trailing edge, the Reynolds-averaged Navier-Stokes (RANS) equations are solved in the flow field. The RANS equations are closed using the one equation Spalart-Allmaras turbulence model, which is iterated using the diagonally dominant alternating direction implicit (DDADI) method. The gradients required by the optimization algorithm (discussed in E below) are solved using an automatic differentiation (AD) adjoint method [15, 16], which efficiently computes gradients with almost no dependence on the number of design variables.

\section{Structural Solver}

The structural solver in the MACH framework is the Toolkit for the Analysis of Composite Structures (TACS) [17]. TACS is a parallel finite-element solver with the capability to handle poorly conditioned problems, which are common in work involving transport aircraft structures. Representation of typical thin-shell wingboxes can lead to matrix condition numbers beyond $\mathcal{O}\left(10^{9}\right)$, which are difficult to solve efficiently and accurately. Through use of a Schurcomplement based parallel direct solver, TACS is able to effectively solve these poorly conditioned problems. As in SUmb, the gradients in TACS are computed using an adjoint method, making the cost of the gradient calculation nearly independent of the number of structural design variables [17]. 


\section{E. Optimization Method}

The calculation of a single aerostructural solution is computationally expensive, making it particularly important that the optimization method used herein uses relatively few function evaluations. Additionally, considering the large number of design variables associated with this aerostructural optimization, it is important that the optimization method is able to efficiently navigate a large-dimensional space. Gradient-free optimization techniques are more likely to converge at a point near the global minimum than gradient-based alternatives. However, the large number of function calls required for gradient free optimizations, particularly involving a sizable number of design variables, makes use of such methods prohibitively expensive given current computing hardware capabilities. As such, a gradient-based method is used, making the efficient gradient calculations discussed earlier important.

The specific algorithm used in this work is SNOPT (sparse nonlinear optimizer) [18]. SNOPT uses a sequential quadratic programming (SQP) approach, making it capable of efficiently handling a problem of the scale of our aerostructural optimization. The Python interface pyOpt [19] is used to interface SNOPT to the problem defined in the MACH framework.

\section{General Problem Summary}

In this section, we provide a context for and an overview of the optimization problems discussed in Section IV. We first describe in more detail the baseline uCRM geometry used as the starting point for all of the optimizations. We also give an overview of the objective functions, design variables, and constraints available in each of the optimizations. The description of each of the optimization problems is given in the next section.

\section{A. uCRM Geometry}

The uCRM jig shape is used as the baseline geometry throughout this work. The uCRM geometry was derived from the NASA Common Research Model (CRM), which has been used by a number of researchers working in computational aerodynamic analysis [20,21] and aerodynamic shape optimization [22]. The CRM geometry is representative of a transonic aircraft similar in size to the Boeing 777. The standard cruise design condition used herein is $M=0.85$ and $C_{L}=0.5$, at $37,000 \mathrm{ft}$. Providing the $1 \mathrm{~g}$ deformed flying shape of the wing is helpful for aerodynamic analysis, as it removes the need to compute the aeroelastic deformations. However, the undeformed jig shape is required for aerostructural optimization. To address this issue, Kenway et al. [7] developed the uCRM using an inverse design procedure that minimized the $L_{2}$ norm of the geometric differences between the CRM and 1-g deflected uCRM. The wingbox used for the internal structure of the UCRM was designed with the intent to closely represent the analogous structure in a Boeing 777. As the CRM has a smaller wingspan and more sweep than the 777, a direct matching of the wingboxes was not possible. Instead, the proportional chord-wise location of the leading and trailing spars at the root and the tip was used to define the corners of the CRM wingbox. The ribs in the CRM wingbox are spaced $28.8 \mathrm{in}(73.15 \mathrm{~cm})$ apart. This approximately matches the rib spacing in the 777 wingbox. The difference in drag between the original CRM and the aerostructurally deformed uCRM is less than one count. The uCRM provides a good starting point for aerostructural optimization, as it represents a reasonably well designed wing that still has room for improvement. The properties of the uCRM are summarized in Table 1.

\section{Trim Correction}

In these analyses, we consider the wing and fuselage configuration, without a tail. As such, a correction needs to be applied to account for the changes in the lift and drag as a result of trimming the aircraft. A surrogate model was used to approximate the effects of the tail, as proposed and validated by Chen $e$ al. [22]. To build this surrogate, Chen performed a lift coefficient constrained sweep of the tail twist angle on the baseline full configuration uCRM. Specifically, a series of tail twist angles were prescribed, and the angle of attack was adjusted to set the lift coefficient to 0.5 . The components of the lift, drag, and pitching moment were then decomposed, so that the effect of the tail in each condition could be isolated and used to construct a model for trim drag.

The model consists of 1-D B-spline interpolations for the lift and drag coefficients, both with respect to the pitching moment required from the tail. Then,

$$
C_{L}=C_{L_{w b}}+C_{L_{t}},
$$

and

$$
C_{D}=C_{D_{w b}}+C_{D_{t}}
$$




\begin{tabular}{lrl}
\hline Parameter & Value & Units \\
\hline Span & 58.8 & $\mathrm{~m}$ \\
Aspect ratio & 9.0 & - \\
Reference wing area & 383.7 & $\mathrm{~m}^{2}$ \\
Reference chord & 7.005 & $\mathrm{~m}$ \\
Moment reference (x) & 33.68 & $\mathrm{~m}$ \\
Moment reference (z) & 4.52 & $\mathrm{~m}$ \\
Leading edge sweep & 37.4 & $\mathrm{deg}$ \\
Maximum take-off weight (MTOW) & 297500 & $\mathrm{~kg}$ \\
Maximum landing weight (MLW) & 213180 & $\mathrm{~kg}$ \\
Maximum zero fuel weight (MZFW) & 195040 & $\mathrm{~kg}$ \\
Operational Empty Weight & 138100 & $\mathrm{~kg}$ \\
Range & 7725 & $\mathrm{~nm}$ \\
Payload & 34000 & $\mathrm{~kg}$ \\
Reserve fuel & 15000 & $\mathrm{~kg}$ \\
Wing weight & 30286 & $\mathrm{~kg}$ \\
Fixed weight & 107814 & $\mathrm{~kg}$ \\
Thrust specific fuel consumption (TSFC) & 0.53 & $\mathrm{lb} /(\mathrm{lbf} \cdot \mathrm{h})$ \\
\hline
\end{tabular}

Table 1: Summary of baseline uCRM parameters based on the uCRM development [7] and publicly available Boeing 777-200ER data [23]

where $C_{L}$ and $C_{D}$ are the approximate trimmed full configuration cruise lift and drag coefficients, $C_{L_{w b}}$ and $C_{D_{w b}}$ are the computed lift and drag coefficients on the wing-body configuration, and $C_{L_{t}}$ and $C_{D_{t}}$ are the lift and drag coefficient contributions produced by the horizontal tail. In this way, we account for the negative lift and positive drag produced by the trimming tail. While this trim penalty model does not predict the lift and drag produced by the tail exactly, there is a close agreement between results produced with this method and results found with a full wing-body-tail optimization [22]. The correction was applied only at cruise, as the maneuver drag is not as important.

\section{B. Objective Function}

The general objective function for the aerostructural optimizations outlined below is a weighted average of the fuel burn and the take-off gross weight (TOGW). These two objectives correlate closely with the aircraft's operating costs and acquisition costs, respectively. Different airlines prefer various compromises between these two costs, and writing the objective as this weighted sum allows us to develop Pareto fronts representing the compromise between the two objectives. The general objective function is written as:

$$
f(x)=\beta \mathrm{FB}+(1-\beta) \text { TOGW }
$$

where FB is the fuel burn, and $\beta \in[0,1]$ is the trade-off variable, defining how the two objectives are weighted.

In these optimizations, a trade-off value of 1 was used, making FB the objective function. The fuel burn for taxi, take-off, climb, and descent are ignored for simplicity. This allows us to compute the fuel consumption over the full mission by applying the Breguet range equation to the design range:

$$
\mathrm{FB}=\mathrm{LGW}\left(\exp \left(\frac{R \mathrm{TSFC}}{V \frac{L}{D}}\right)-1\right),
$$

where LGW is the landing gross weight, $R$ is the design range, TSFC is the thrust specific fuel consumption, $V$ is the cruise speed, and $\frac{L}{D}$ is the lift-to-drag ratio. The lift-to-drag ratio is computed using the trim corrected lift and drag coefficients, with an additional 50 drag counts added to model the losses from the vertical tail, nacelles, and pylons, which are not explicitly modeled with CFD.

\section{Design Variables}

We consider the design variables in three sets: aerodynamic, geometric, and structural variables. The only aerodynamic design variables are the angles of attack at each flight condition, which are added to assure that a lift constraint can 


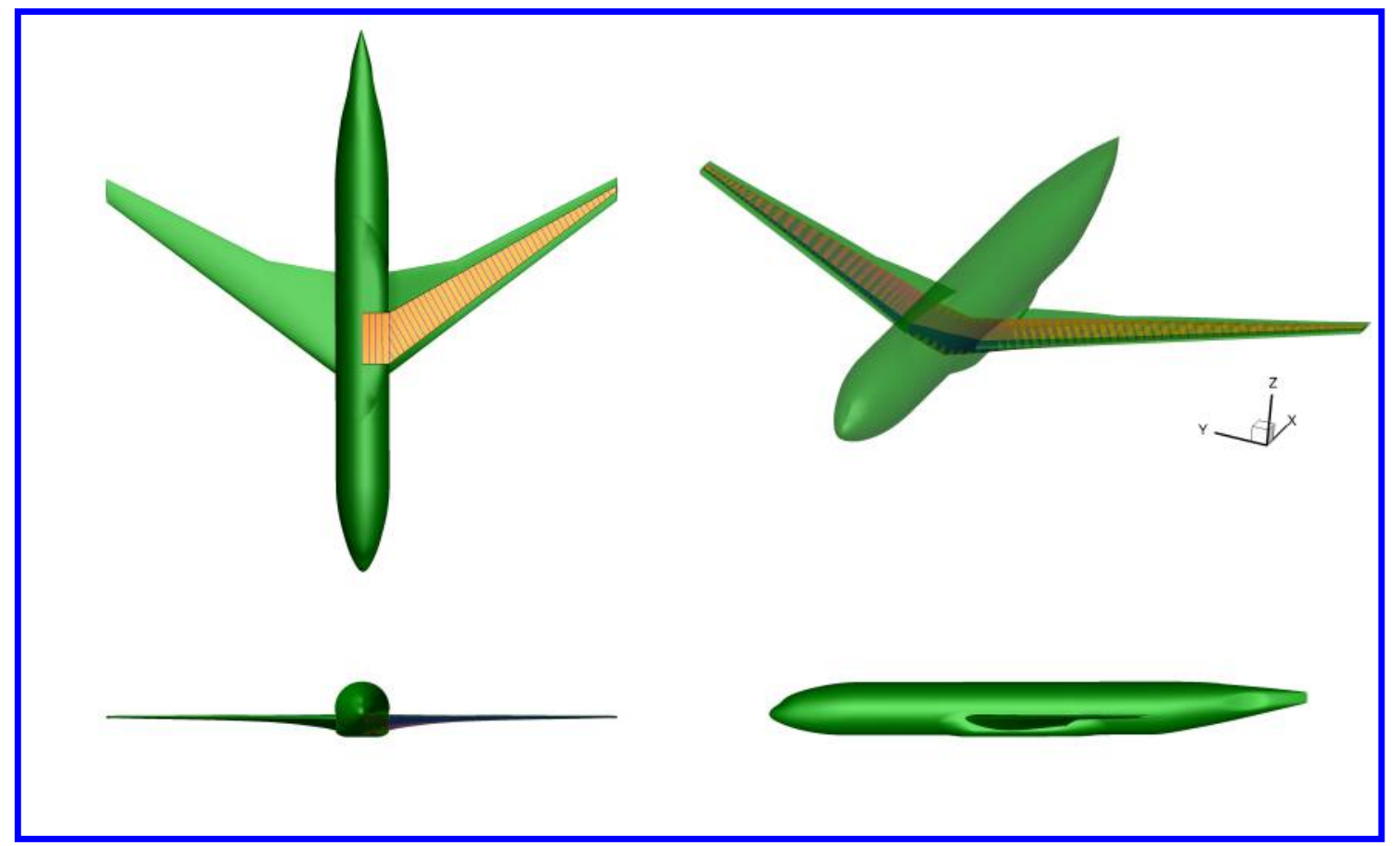

Figure 1: The baseline wing-body uCRM jig shape and wingbox.

be added without making the problem ill-posed. Eight twist variables and the z-deflections of 192 control points prescribe aerodynamic shape changes. The control points are not given freedom in the $x$ and $y$ directions, meaning that the planform of the wing is held fixed. To achieve configuration-specific morphing, a subset of the control points is added a second time as design variables. These 80 control points, all of which are on the aft $40 \%$ of the wing, behind the wing box, are associated only with a specific configuration, and are not applied to the FFD during the analysis of alternate configurations. The subset of control points associated with the morphing trailing edge are shown in blue in Figure 2.

Structural design variables are used to parametrize the geometry of the wingbox. Note that in these analyses, the full wingbox, including the center section spanning the fuselage, is considered. This means that the structural deflection at the wing-fuselage interface is not necessarily zero. Large interface deflections result in mesh tears, but the optimization permits small deformations. A smeared stiffness panel approach is used in the analysis of the structural deformations. The geometry of the stiffened panels is shown in Figure 3. To simplify the model and reduce the number of design variables, we take $w_{b}=h_{s}$ and $t_{b}=t_{w}$. This yields four design variables for the skins and spars in each bay of the wingbox: panel thickness, panel length, stiffener thickness, and stiffener height. The panel length is included as a structural design variable to simplify buckling calculations, and is constrained to be consistent with the geometric design variables through a series of constraints. Four additional design variables are used to define the pitch of the stiffeners on the skins and spars. Note that the stiffener pitch is taken as constant on each of these components. All together, this yields 884 structural design variables.

In summary, there are 884 structural variables, an angle of attack, 8 twist variables, 192 shape variables spanning the entire wing, and 80 morphing shape variables on the aft $40 \%$ of the wing. To assure the well-posedness of the problem, given that there are lift, stress, and buckling constraints as discussed in Subsection D, the structural and aerodynamic design variables are included in each of the optimizations. The selection of aerodynamic design variables defines the different optimizations discussed below. For the retrofitted wing, the only aerodynamic design variables available to the optimizer are those representing the morphing trailing edge. Note that this adds 80 design variables for each of the flight configurations, allowing the wing to have alternate morphed shapes for cruise and maneuver. As such, there are 160 aerodynamic design variables used in the retrofitted optimization. In the clean sheet design, 280 aerodynamic variables are used. The 8 twist and 192 full wing shape variables are applied at each flight condition, and at the off design maneuver condition, one set of 80 morphing variables is used in conjunction with the full wing shape 


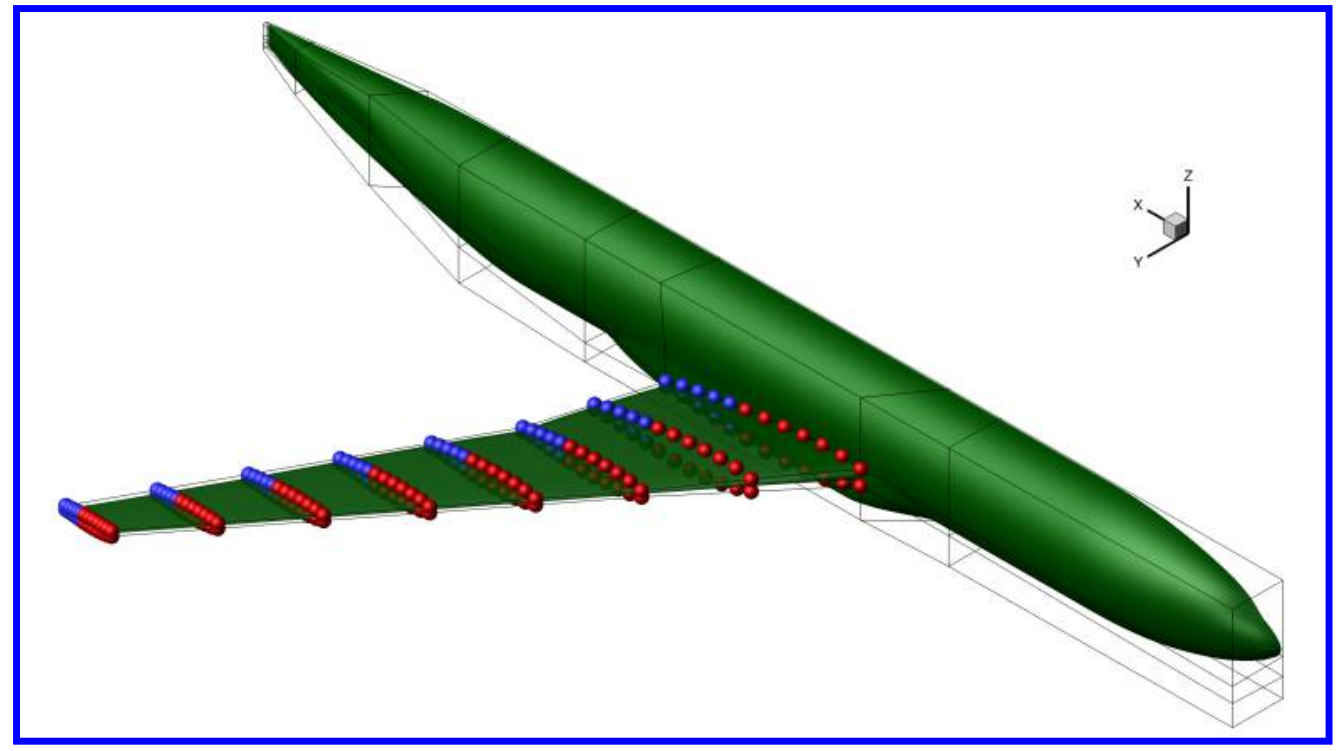

Figure 2: Each sphere represents one of the 192 FFD control points. The blue spheres are the subset of control points associated with the morphing trailing edge.

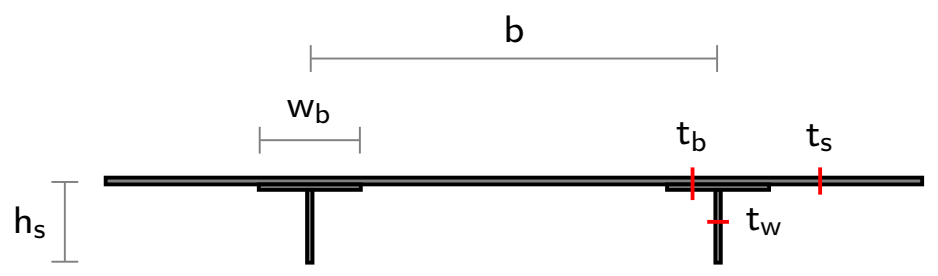

Figure 3: The structural design variables parametrize the geometry as has been done in past work [7].

variables.

\section{Constraints}

To assure the optimization results represent a physically feasible system, a number of constraints are added to the problem. The optimizations below consider flight at two conditions: cruise and maneuver. The first constraints give steady flight at these two conditions, as the lift is set equal to the weight times the load factor. A $2.5 \mathrm{~g}$ load factor is used at maneuver. More details about the two flight conditions can be found in Table 2

\begin{tabular}{lrr}
\hline Parameter & Cruise & Maneuver \\
\hline Load factor & 1.0 & 2.5 \\
Mach number & 0.85 & 0.64 \\
Altitude (ft.) & 37000 & 0 \\
Weight & $\frac{1}{2}($ TOGW + LGW $)$ & TOGW \\
\hline
\end{tabular}

Table 2: Overview of the cruise and maneuver flight conditions.

The first geometric constraint prevents the wing volume from decreasing, assuring that there is sufficient space in the wing for fuel. The airfoil thickness is also constrained not to decrease at a number of locations. The leading edge thickness is constrained to prevent the optimizer from designing sharp leading edges, which perform poorly at low speeds. The trailing edge thickness is constrained to maintain a manufacturable trailing edge. Finally, the thickness at 
the aft spar of the wingbox is constrained not to decrease, to assure there is sufficient space for control surface actuators. Constraints are added to the leading and trailing edges at cruise to prevent shearing twist. These constraints require that the control points along the front and back faces of the FFD move in equal and opposite directions, such that the center of the FFD faces won't be moved by shape changes. The structural constraints are used to prevent failure, maintain consistency, and again prevent unrealistic designs. Adjacency constraints prevent unreasonable changes in the skins and stiffeners between neighbouring panels. Nonlinear consistency constraints are used to constrain panel lengths to match the length prescribed by the FFD, as discussed in Subsection C. Finally, to assure the structure is sufficiently robust, buckling and stress constraints are added for the $2.5 \mathrm{~g}$ maneuver case. These constraints are aggregated into 6 KS constraints.

\section{Optimization Results}

In this section, we look to quantify the fuel burn benefit resulting from a morphing trailing edge. We do so by comparing an optimized wing without a morphing trailing edge to a wing retrofitted with a morphing TE and a wing designed with a morphing TE. Before considering the comparison between these solutions, we consider an overview of the optimization problem for the baseline aerostructural optimization, as given in Table 3.

\begin{tabular}{rlll}
\hline & Function/variable & Description & Quantity \\
\hline minimize & Fuel burn & & \\
with respect to & $x_{\alpha_{\mathrm{c}}}$ & Angle of attack at cruise & 1 \\
& $x_{\alpha_{\mathrm{m}}}$ & Angle of attack at maneuver & 1 \\
& $x_{\text {shape }}$ & Full wing FFD control points & 192 \\
& $x_{\text {twist }}$ & Wing twist & 8 \\
& $x_{\text {struct }}$ & Structural sizing variables & 884 \\
& & Total DVs & $\mathbf{1 0 8 6}$ \\
subject to & $L=n_{i} W$ & Lift & 2 \\
& $V / V_{\text {init }} \geq 1$ & Fuel volume & 1 \\
& $t /\left.t_{\text {init }}\right|_{\mathrm{LE}} \geq 1$ & Leading edge thickness & 20 \\
& $t /\left.t_{\text {init }}\right|_{\mathrm{TE}} \geq 1$ & Trailing edge thickness & 50 \\
& $t /\left.t_{\text {init }}\right|_{\text {spar }} \geq 1$ & Aft spar thickness constraint & 20 \\
& $\Delta z_{\mathrm{LE}_{\text {upper }}}=-\Delta z_{\mathrm{LE}_{\text {lower }}}$ & Fixed leading edge & 8 \\
& $\Delta z_{\mathrm{TE}_{\text {upper }}}=-\Delta z_{\mathrm{TE}_{\text {lowe }}}$ & Fixed trailing edge & 8 \\
& $L_{\text {panel }}-x_{\text {panel }}=0$ & Non-linear consistency constraints & 302 \\
& $K S_{\text {stress }} \leq 1$ & Maneuver stress & 3 \\
& $K S_{\text {buckling }_{1}} \leq 1$ & Maneuver buckling & 3 \\
& $\left|x_{\text {struct }_{i}}-x_{\text {struct }}{ }_{i+1}\right| \leq 0.0005$ & Linear adjacency constraints & 696 \\
& Total constraints & $\mathbf{1 1 1 3}$ \\
\hline
\end{tabular}

Table 3: Overview of the baseline uCRM aerostructural optimization problem.

The results for this baseline aerostructural optimization are given as a reference in each of the following sections. Additionally, the aerostructural optimization problems defining the retrofit and clean sheet cases will be described in relation to this baseline optimization, as there are only small changes to the optimization problem in each case.

\section{A. Retrofit Wing}

In our first study, we consider the benefits associated with retrofitting a morphing trailing edge device onto an existing wing. To do this, we compare the aerostructural performance of the optimized uCRM to that of the uCRM enhanced with design variables in the morphing section of the wing. This optimization varies from the baseline optimization in the following ways:

- The 192 full wing FFD control points are not used

- 80 control points are added to control the morphing section, both at cruise and maneuver (adding 160 DVs total)

- The 8 twist design variables are not included 


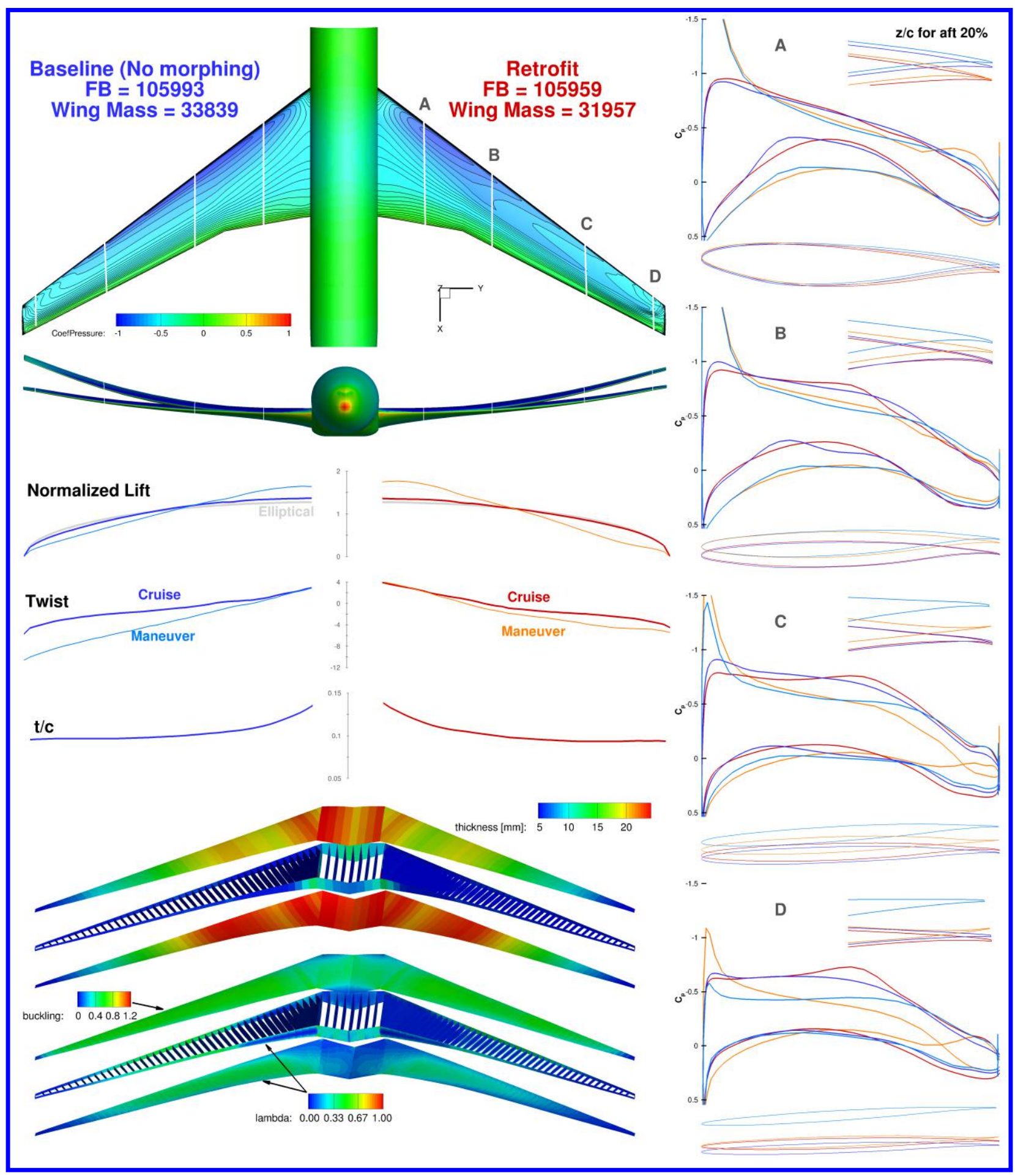

Figure 4: A comparison of a baseline aerostructural optimization of the uCRM to an optimization of the baseline uCRM retrofitted with a morphing trailing edge. The morphing TE produced an airfoil with reversed camber at the outboard sections during maneuver, helping to shift the loads inboard. 
- The fixed trailing edge constraint is removed

A comparison of the resulting wing shapes is given in Figure 4.

When comparing the two cases, we see that the objective fuel burn values are nearly equal, varying by $0.032 \%$. Interestingly, the baseline uCRM produces a fuel burn of $112491 \mathrm{~kg}$, which means that both optimizations were able to reduce the fuel burn by approximately 5.8\%. Looking at the pressure coefficient contours of the two wings at cruise, we see that the baseline optimization produces (desirable) nearly parallel pressure contours, while the retrofit can produce such parallel contours only on the aft section of the wing. This feature of the pressure contours matches expectations, as the retrofit wing does not have shape control of the front portion of the wing. Interestingly, the retrofit wing is still able to achieve the same level of fuel burn reduction, due largely to its wing mass reduction. The reduction in the wing mass is achieved through load alleviation at the maneuver condition, which allows the structural member thicknesses to be decreased. The lift distributions of the two cases show a more substantial inboard shift of loading achieved in the retrofit case. Correspondingly, from the front view, it is clear that the wing deflection at maneuver is reduced through the use of the morphing TE.

Further insights about the outboard load alleviation achieved by the morphing can be found by considering the slice information on the right of Figure 4. At sections C and D, we see that the morphing has reversed the airfoil's camber near the TE for the maneuver case. Correspondingly, the sliced pressure distributions show a region of negative lift is produced towards the trailing edge tip. Interestingly, this effect also contributes to a change in the twist distribution between the two cases at maneuver. In the baseline case, passive load alleviation results from structural washout, as evidenced by the twist distribution. The twist distribution for the retrofit wing at maneuver is much closer to that at cruise. This result illustrates the difference in the two methods of load alleviation: passive load alleviation via aeroelastic tailoring to produce washout at maneuver, and active load alleviation via airfoil morphing, producing negative chamber regions near the tip of the wing. As a final interesting note, the thickness distributions for the two cases is very similar. The retrofit wing is very slightly thicker near the root and thinner near the tip, but this difference is much smaller than the other differences between the two configurations, suggesting the addition of the morphing trailing edge has little effect on the ideal thickness distribution.

\section{B. Clean Sheet Design}

We now consider the benefits of the morphing trailing edge on a clean sheet design, and again compare to an optimized wing without morphing. The only difference between the baseline optimization and the optimization of the clean sheet design is the addition of 80 morphing design variables at the maneuver case. The comparison between the two results is shown in Figure 5.

The fuel burn for the clean sheet design is $0.36 \%$ lower than that of the baseline, non-morphing optimized design. This improvement is slightly more than that of the retrofit wing. The cruise pressure coefficient contours show the advantage of having control of shape design variables which encompass the entire wing. The contours of the baseline and clean sheet wings at cruise are nearly identical, and both match what is expected for an optimized wing at its design condition. The lift distribution at cruise shows that the clean sheet design is able to match an elliptical distribution more closely than the baseline case. Given that the only difference between the two cases is the inclusion of morphing variables at maneuver, it follows that this improvement in the cruise performance is a result of the decrease in coupling between the cruise and maneuver configurations. The inclusion of morphing at maneuver removes the need to trade-off between cruise performance and maneuver feasibility, but in the baseline optimization, consideration of performance at maneuver can compromise cruise performance, giving lift distributions slightly different than the elliptic ideal. The twist and thickness distributions show similar trends to those from the retrofit design, although the clean sheet maneuver twist does not follow the cruise twist as closely as in the retrofit case.

The structural thickness distribution shows the thicknesses of the structural members to be much closer than they were in the comparison of the baseline to the retrofit wing. This result is consistent with the wing mass comparison as well. Looking at the lift distribution of the clean sheet design at maneuver, we again see that it has achieved more load alleviation than the baseline optimization, however there is not as much load alleviation as was present in the retrofit wing. The airfoil and pressure coefficient slices suggest a similar conclusion, as the clean sheet design also reversed the camber of the outboard airfoils, but did so to a much lower extent than the retrofit wing. The region of negative lift is confined to a smaller area near the trailing edge tip. This is interesting, given that the clean sheet design used the same set of morphing control points as the retrofit case.

From these results, we conclude that the morphing trailing edge produces two methods by which the optimizer can reduce the fuel burn. First, improved load alleviation at maneuver shifts the critical loads further inboard, resulting in a lighter structural weight. The second method involves the weakening of the coupling between performance 


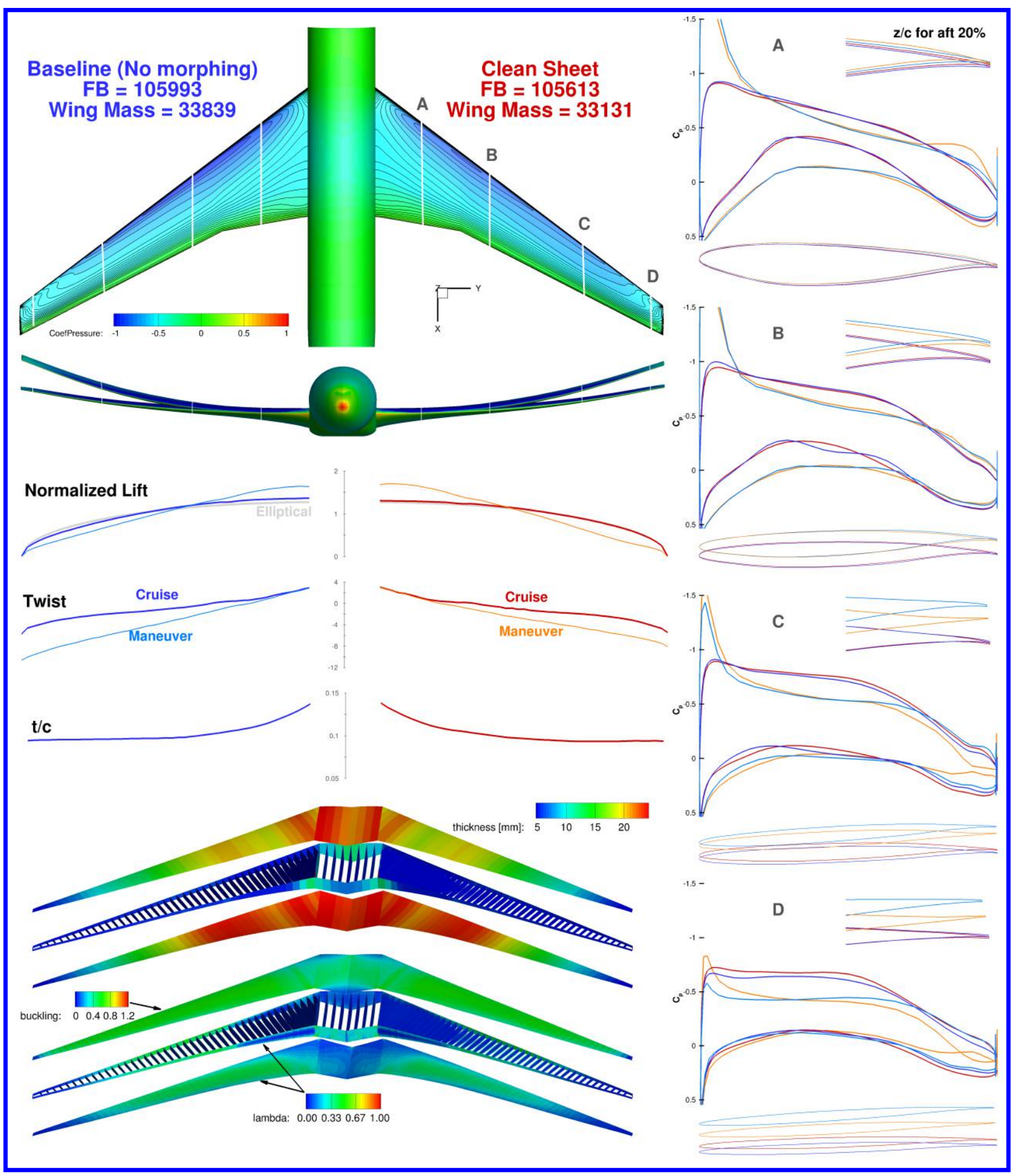

Figure 5: A comparison of a baseline aerostructural optimization of the uCRM to an analogous optimization of a clean sheet wing design with a morphing trailing edge. The morphing trailing edge is able to reduce the lift produced on the outboard section, shifting loads inboard at maneuver to alleviate stresses on the wingbox. 
at various flight conditions. The coupling is not completely removed, but the adaptability of the morphing trailing edge at off-design conditions allows the design at each condition to focus more on its own objectives, with less influence from the other flight conditions. This means that there are fewer compromises and trade-offs needed at each flight condition, which yields better performance. In the retrofit design, the aerodynamic improvements available at the cruise conditions were limited, as the twist distribution and much of the airfoil shape could not be changed, so the optimizer focused on improving the fuel burn through the first method. This is apparent as the wing mass is significantly decreased in the retrofit case. In the clean sheet design, both methods are used, as the cruise lift distribution more closely follows the ideal distribution, and the maneuver distribution is shifted further inboard than that of the non-morphing wing, however the wing mass is reduced less than it was for the retrofit case.

\section{Conclusion}

We simulated the design process for a standard non-morphing wing, and wing retrofitted with a morphing trailing edge, and a clean sheet wing designed with the morphing trailing edge. Compared to the starting point of the optimizations, the baseline optimized wing reduced the fuel burn by $5.8 \%$. The same level of fuel burn reduction was achieved in the retrofit optimization, where the optimizer had no control over the twist distribution or the shape variables on the front $60 \%$ of the wing. The clean sheet optimized wing reduced the fuel burn by $6.1 \%$ compared to the uCRM starting configuration. The morphing trailing edge was found to achieve fuel burn reductions via two mechanisms. The first is the ability to improve maneuver load alleviation, allowing for lighter wing structures. The second is a reduction of the coupling between the cruise and maneuver cases, which allows the cruise configuration to improve without causing adverse effects on maneuver performance. The clean sheet design used both of these mechanisms, while the retrofit design focused on reducing the weight of the wing, as the increases it could make at cruise were limited due to its design space.

These results show that a morphing trailing edge has the potential to decrease the fuel burn, but the improvements with respect to an aerostructurally optimized standard wing were rather marginal. This is due largely to the problem formulation. Given the geometric constraints and the set of two flight conditions, only one of which was concerned with the aerodynamic performance (in terms of $L / D$ ), the potential savings from using a morphing trailing edge were limited. To give a more representative comparison in future work, planform variables and additional flight conditions could be added to the problem.

\section{Acknowledgements}

This work is supported by NASA through award No. NNX11AI19A. This work used the Extreme Science and Engineering Discovery Environment (XSEDE), which is supported by National Science Foundation grant number ACI-1053575 [24]. 


\section{References}

-[1] Kota, S., Hetrick, J., Osborn, R., Paul, D., Pendleton, E., Flick, P., and Tilmann, C., "Design and Application of Compliant Mechanisms for Morphing Aircraft Structures," Proceedings of SPIE, Vol. 5054, 2003, p. 25.

[2] Kota, S., Osborn, R., Ervin, G., Maric, D., Flick, P., and Paul, D., "Mission Adaptive Compliant Wing-Design, Fabrication and Flight Test," RTO Applied Vehicle Technology Panel (AVT) Symposium, 2009.

-[3] Sofla, A. Y. N., Meguid, S. A., Tan, K. T., and Yeo, W. K., "Shape Morphing of Aircraft Wing: Status and Challenges," Materials \& Design, Vol. 31, No. 3, 3 2010, pp. 1284-1292. doi:10.1016/j.matdes.2009.09.011.

- [4] Molinari, G., Quack, M., Dmitriev, V., Morari, M., Jenny, P., and Ermanni, P., "Aero-Structural Optimization of Morphing Airfoils for Adaptive Wings,” Journal of Intelligent Material Systems and Structures, Vol. 22, No. 10, 2011, pp. 1075-1089. doi:10.1177/1045389X11414089.

[5] Lyu, Z., Kenway, G. K. W., and Martins, J. R. R. A., "RANS-based Aerodynamic Shape Optimization Investigations of the Common Research Model Wing," Proceedings of the AIAA Science and Technology Forum and Exposition (SciTech), National Harbor, MD, January 2014. doi:10.2514/6.2014-0567, AIAA-2014-0567.

[6] Lyu, Z. and Martins, J. R. R. A., "Aerodynamic Shape Optimization of an Adaptive Morphing Trailing Edge Wing," Proceedings of the 15th AIAA/ISSMO Multidisciplinary Analysis and Optimization Conference, Atlanta, GA, June 2014, AIAA 2014-3275.

[7] Kenway, G. K. W., Kennedy, G. J., and Martins, J. R. R. A., "Aerostructural Optimization of the Common Research Model Configuration," 15th AIAA/ISSMO Multidisciplinary Analysis and Optimization Conference, Atlanta, GA, June 2014, AIAA 2014-3274.

- [8] Kenway, G. K. W., Kennedy, G. J., and Martins, J. R. R. A., "Scalable Parallel Approach for High-Fidelity SteadyState Aeroelastic Analysis and Adjoint Derivative Computations," AIAA Journal, Vol. 52, No. 5, 2014, pp. 935-951. doi:10.2514/1.J052255.

- [9] Kenway, G. K. W. and Martins, J. R. R. A., "Multipoint High-fidelity Aerostructural Optimization of a Transport Aircraft Configuration,” Journal of Aircraft, Vol. 51, No. 1, 2014, pp. 144-160. doi:10.2514/1.C032150.

[10] Liem, R. P., Kenway, G. K. W., and Martins, J. R. R. A., "Multimission Aircraft Fuel Burn Minimization via Multipoint Aerostructural Optimization,” AIAA Journal, 2014. doi:10.2514/1.J052940, (In press).

-[11] Kennedy, G. J. and Martins, J. R. R. A., “A parallel aerostructural optimization framework for aircraft design studies,” Structural and Multidisciplinary Optimization, 2014. doi:10.1007/s00158-014-1108-9.

[12] Kenway, G. K., Kennedy, G. J., and Martins, J. R. R. A., "A CAD-Free Approach to High-Fidelity Aerostructural Optimization," Proceedings of the 13th AIAA/ISSMO Multidisciplinary Analysis Optimization Conference, Fort Worth, TX, Sept. 2010, AIAA 2010-9231.

[13] Uyttersprot, L., Inverse Distance Weighting Mesh Deformation, Ph.D. thesis, Delft University of Technology, 2014.

[14] van der Weide, E., Kalitzin, G., Schluter, J., and Alonso, J. J., "Unsteady Turbomachinery Computations Using Massively Parallel Platforms," Proceedings of the 44th AIAA Aerospace Sciences Meeting and Exhibit, Reno, NV, 2006, AIAA 20060421.

[15] Mader, C. A., Martins, J. R. R. A., Alonso, J. J., and van der Weide, E., “ADjoint: An Approach for the Rapid Development of Discrete Adjoint Solvers,” AIAA Journal, Vol. 46, No. 4, April 2008, pp. 863-873. doi:10.2514/1.29123.

-[16] Lyu, Z., Kenway, G. K., Paige, C., and Martins, J. R. R. A., "Automatic Differentiation Adjoint of the Reynolds-Averaged Navier-Stokes Equations with a Turbulence Model," 21st AIAA Computational Fluid Dynamics Conference, San Diego, CA, Jul 2013. doi:10.2514/6.2013-2581.

-[17] Kennedy, G. J. and Martins, J. R. R. A., "A Parallel Finite-Element Framework for Large-Scale Gradient-Based Design Optimization of High-Performance Structures," Finite Elements in Analysis and Design, Vol. 87, September 2014, pp. 56-73. doi:10.1016/j.finel.2014.04.011.

[18] Gill, P. E., Murray, W., and Saunders, M. A., “SNOPT: An SQP Algorithm for Large-Scale Constrained Optimization,” SIAM journal on optimization, Vol. 12, No. 4, 2002, pp. 979-1006. doi:10.1137/S1052623499350013.

- [19] Perez, R. E., Jansen, P. W., and Martins, J. R. R. A., "pyOpt: a Python-Based Object-Oriented Framework for Nonlinear Constrained Optimization," Structural and Multidisciplinary Optimization, Vol. 45, No. 1, January 2012, pp. 101-118. doi:10.1007/s00158-011-0666-3.

[20] Vassberg, J. C., DeHaan, M. A., Rivers, S. M., and Wahls, R. A., "Development of a Common Research Model for Applied CFD Validation Studies," 2008, AIAA 2008-6919.

[21] Vassberg, J., "A Unified Baseline Grid about the Common Research Model Wing/Body for the Fifth AIAA CFD Drag Prediction Workshop (Invited)," 29th AIAA Applied Aerodynamics Conference, Jul 2011. doi:10.2514/6.2011-3508.

[22] Chen, S., Lyu, Z., Kenway, G. K. W., and Martins, J. R. R. A., "Aerodynamic Shape Optimization of the Common Research Model," AIAA Science and Technology Forum and Exposition (SciTech), Kissimmee, FL, January 2014, AIAA-2015-1718. 
[23] Anonymous, “777-200/300 Airplane Characteristics for Airport Planning,” Tech. Rep. D6-58329, Boeing Commercial Airplanes, July 1998.

[24] John Towns, Timothy Cockerill, M. D. I. F. K. G. A. G. V. H. S. L. D. L. G. D. P. R. R. J. R. S. and Wilkens-Diehr, N., "XSEDE: Accelerating Scientific Discovery," Computing in Science and Engineering, Vol. 16, No. 5, 2014, pp. 62-74. doi:10.1109/MCSE.2014.80. 


\section{This article has been cited by:}

1. Pedro F. Albuquerque, Pedro V. Gamboa, Miguel A. Silvestre. Mission-Based Multidisciplinary Aircraft Design Optimization Methodology Tailored for Adaptive Technologies. Journal of Aircraft, ahead of print1-16. [Abstract] [Full Text] [PDF] [PDF Plus]

2. Timothy R. Brooks, Gaetan K. Kenway, Joaquim Martins. Undeflected Common Research Model (uCRM): An Aerostructural Model for the Study of High Aspect Ratio Transport Aircraft Wings . [Citation] [PDF] [PDF Plus]

3. David A. Burdette, Gaetan K. Kenway, Joaquim Martins. Performance Evaluation of a Morphing Trailing Edge Using Multipoint Aerostructural Design Optimization . [Citation] [PDF] [PDF Plus]

4. Sridhar Kota, Peter Flick, Fayette S. Collier. Flight Testing of FlexFloilTM Adaptive Compliant Trailing Edge . [Citation] [PDF] [PDF Plus]

5. Zhoujie Lyu, Joaquim R. R. A. Martins. 2015. Aerodynamic Shape Optimization of an Adaptive Morphing Trailing-Edge Wing. Journal of Aircraft 52:6, 1951-1970. [Abstract] [Full Text] [PDF] [PDF Plus] 